\title{
Zero-range potential in the model of the spinor field inter- acting with a material plane: conditions for transparency of the plane to Dirac particles
}

\author{
Yu. M. Pismak ${ }^{1, *}$ \\ ${ }^{1}$ Saint-Petersburg State University, Ulyanovskaya street 1, Petrodvorets, 198504 St-Petersburg, Russia
}

\begin{abstract}
In the model of interaction of a spinor field with a homogeneous isotropic material plane, the possibility of the passage of a Dirac particle through it without reflection and a change in polarization is studied. The model was proposed earlier in the framework of the Symanzik approach to describe the interaction of spinor quantum fields with two-dimensional materials. In its framework the scattering processes of Dirac particles and the properties of their bound states were investigated. In this paper, we study the conditions under which the plane is completely transparent to the particles. A description of the region of model parameters characterizing the properties of the plane material is obtained explicitly and given in the article, as well as expressions in terms of these parameters for the momentum and polarization of the particle at which interaction with the plane does not affect its movement.
\end{abstract}

\section{Introduction}

Models with zero radius potentials have been considered in the articles of many authors. They arise naturally in models of interaction of quantized fields with space-time inhomogeneities of dimension less than four [1]. They were used, in particular, in the models of interaction of fields of quantum electrodynamics [2] (QED) with two-dimensional (2D) material objects constructed within the framework of the Symanzik approach [3-11]. In these models, the Casimir forces and the characteristics of the processes of scattering of photons and Dirac particles were calculated, and the properties of bound states were also studied [3-11]. The aim of our work is to investigate the possibilities of special processes in the interaction of Dirac particles with a 2D-material in which their dynamics do not differ from the dynamics of free particles and in this sense the material object turns out to be transparent.

The main idea used to model the interaction of QED fields with a 2D-material, was to modify the usual action functional of QED by adding an additional contribution (defect action) describing the effect of a macro object on quantum fluctuations of the electromagnetic vector field $A$ and Dirac spinor fields $\bar{\psi}, \psi$. The requirements of locality, gauge invariance and renormalizability significantly restrict the possibilities of constructing a model. If the shape of a two-dimensional object is described by the equation $\Phi(x)=0$, where $x$ is the point of the (3+1)-space-time, then the defect action functional $S_{d e f}(\bar{\psi}, \psi, A, \Phi)$ with the maximal

\footnotetext{
*e-mail: ypismak@gmail.com
} 
number of parameters in such a theory is the sum of three terms concentrated in the subspace $\Phi(x)=0$ of the full 4-dimensional space-time:

$$
S_{d e f}(\bar{\psi}, \psi, A, \Phi)=S_{d e f}(A, \Phi)+S_{d e f}(\bar{\psi}, \psi, \Phi)+S_{d e f}(\Phi) .
$$

Here, $S_{d e f}(A, \Phi)$ is the Chern-Simons action functional

$$
S_{d e f}(A, \Phi)=\frac{a}{2} \int \varepsilon^{\lambda \mu \nu \rho} \partial_{\lambda} \Phi(x) A_{\mu}(x) F_{v \rho}(x) \delta(\Phi(x)) d^{4} x
$$

with totally antisymmetric tensor $\varepsilon^{\lambda \mu \nu \rho}\left(\varepsilon^{0123}=1\right)$ and a dimensionless coupling constant $a$. Then the most general form of the spinor defect action $S_{\text {def }}(\bar{\psi}, \psi, \Phi)$ is

$$
S_{d e f}(\bar{\psi}, \psi, \Phi)=\sum_{j=1}^{16} \int \alpha_{j} \bar{\psi}(x) \Gamma_{j} \psi(x) \delta(\Phi(x)) d^{4} x
$$

where $\Gamma_{j}$ are the 16 basis Dirac matrices and $\alpha_{j}$ are dimensionless coupling constants. The defect action $S_{d e f}(\Phi)$ does not depend on the QED fields and is necessary for cancellation of ultraviolet divergences in renormalized Casimir energy density.

Symmetry requirements can essentially reduce the number of parameters in the model. So in the model of interaction of QED fields with a homogeneous isotropic plane $x^{3}=0$, which was considered in [3], [4], the spinor action is written in the form

$$
S(\bar{\psi}, \psi)=\int \bar{\psi}(x)\left(i \hat{\partial}-m+Q \delta\left(x^{3}\right)\right) \psi(x) d x
$$

with dimensionless matrix $Q$ of the form

$$
Q=r_{1} \mathbf{1}+i r_{2} \gamma_{5}+r_{3} \gamma_{3}+r_{4} \gamma_{5} \gamma_{3}+r_{5} \gamma_{0}+r_{6} \gamma_{5} \gamma_{0}+i r_{7} \gamma_{0} \gamma_{3}+i r_{8} \gamma_{1} \gamma_{2}
$$

describing the coupling of Dirac fields with the material plane. Here, $r_{1} \ldots r_{8}$ are real constant parameters, $i=\sqrt{-1}, \mathbf{1}$ is the unit $4 \times 4$ matrix, $\gamma^{j}, j=0,1,2,3$ are the Dirac matrices and $\gamma^{5}=i \gamma^{0} \gamma^{1} \gamma^{2} \gamma^{3}$. In this paper, we use this model for investigating the problem of transparency of the material plane for Dirac particles. We show that under certain conditions for a plane wave of the spinor field, the interaction of which with the plane $x^{3}=0$ is described by the Zero-range potential $Q \delta\left(x_{3}\right)$, the plane is transparent.

\section{Statement of the problem}

Let us introduce some the convenient notations. We denote

$$
\bar{x}=\left(x_{0}, x_{1}, x_{2}\right), \overline{p x}=p_{0} x_{0}-p_{1} x_{1}-p_{2} x_{2}, \hat{\bar{p}}=\overline{\gamma p} .
$$

For $2 \times 2$-matrix $\mathrm{M}$ with elements $M_{i j}, i, j=1,2$, we define the $4 \times 4$-matrices $M^{(+)}, M^{(-)}$ in the following way

$$
M^{(+)}=\left(\begin{array}{cccc}
M_{11} & 0 & M_{12} & 0 \\
0 & 0 & 0 & 0 \\
M_{21} & 0 & M_{22} & 0 \\
0 & 0 & 0 & 0
\end{array}\right), M^{(-)}=\left(\begin{array}{cccc}
0 & 0 & 0 & 0 \\
0 & M_{11} & 0 & M_{12} \\
0 & 0 & 0 & 0 \\
0 & M_{21} & 0 & M_{22}
\end{array}\right) .
$$

For arbitrary matrices $M_{1}^{( \pm)}, M_{2}^{(\mp)}$,

$$
M_{1}^{( \pm)} M_{2}^{(\mp)}=0, M_{1}^{( \pm)} M_{2}^{( \pm)}=M_{3}^{( \pm)}
$$


If we denote the unit $2 \times 2$ - matrix as $\tau_{0}$, the Pauli matrices as $\tau_{1}, \tau_{2}, \tau_{3}$, and the corresponding $4 \times 4$-matrices as $\tau_{j}^{(+)}, \tau_{j}^{(-)}, j=0,1,2,3$, then these matrices fulfill the commutation relations

$$
\tau_{j}^{( \pm)} \tau_{k}^{( \pm)}+\tau_{k}^{( \pm)} \tau_{j}^{( \pm)}=\delta_{j k} \tau_{0}^{( \pm)}, \tau_{j}^{( \pm)} \tau_{k}^{( \pm)}-\tau_{k}^{( \pm)} \tau_{j}^{( \pm)}=2 i \sum_{l=1}^{3} \epsilon_{j k l} \tau_{l}^{( \pm)}, j, k=1,2,3,
$$

with fully antisymmetric tensor $\epsilon_{j k l}, \epsilon_{123}=1$, and the matrix $Q$ can be presented as

$$
Q=Q^{(+)}+Q^{(-)}, Q^{( \pm)}=\sum_{j=0}^{3} q_{j}^{( \pm)} \tau_{j}^{( \pm)}
$$

where $q_{0}^{( \pm)}=r_{18}^{ \pm}, q_{1}^{( \pm)}=i r_{27}^{ \pm}, q_{2}^{( \pm)}= \pm i r_{36}^{\mp}, q_{3}^{( \pm)}=\mp r_{45}^{\mp}$, and $r_{i j}^{ \pm}=r_{i} \pm r_{j}$.

The action functional (1) generates a modified Dirac equation of the form

$$
\left(i \hat{\partial}-m+Q \delta\left(x^{3}\right)\right) \psi(x)=0
$$

In accordance with the task formulated by us, we are looking for a solution to this equation in the form of a plane wave

$$
\psi(x, \bar{p})=e^{-i p x} \psi(\bar{p}), p_{3}=\sqrt{\bar{p}^{2}-m^{2}} .
$$

This means that despite the interaction with the plane, which is described in (3) by the matrix $\mathrm{Q}$, the wave function of the particle satisfies the usual Dirac equation of the theory without interaction - that is, the particle is realized as free. $\psi(\bar{p})$ :

Substituting the spinor $\psi(x, \bar{p})$ in the equation (3), we obtain two equations for the spinor

$$
(\hat{p}-m) \psi(\bar{p})=0, Q \psi(\bar{p})=0 .
$$

The first of them is the usual free Dirac equation [2] for the spinor in the momentum representation. It has two linearly independent solutions for $p_{0}>0$ (Dirac particles)

$$
\psi_{1}(\bar{p})=\left\{\begin{array}{c}
1 \\
0 \\
\frac{-p_{3}}{m+p_{0}} \\
\frac{-p_{1}-i p_{2}}{m+p_{0}}
\end{array}\right\}, \psi_{2}(\bar{p})=\left\{\begin{array}{c}
0 \\
1 \\
\frac{-p_{1}+i p_{2}}{m+p_{0}} \\
\frac{p_{3}}{m+p_{0}}
\end{array}\right\}, p_{3}=\sqrt{\bar{p}^{2}-m^{2}}
$$

and for $p_{0}<0$ (Dirac antiparticles)

$$
\psi_{1}^{\prime}(\bar{p})=\left\{\begin{array}{c}
\frac{p_{1}-i p_{2}}{m-p_{0}} \\
\frac{p_{3}}{p_{0}-m} \\
0 \\
1
\end{array}\right\}, \psi_{2}^{\prime}(\bar{p})=\left\{\begin{array}{c}
\frac{p_{3}}{m-p_{0}} \\
\frac{p_{1}+i p_{2}}{m-p_{0}} \\
1 \\
0
\end{array}\right\}, \quad p_{3}=\sqrt{\bar{p}^{2}-m^{2}}
$$

Thus, the spinor $\psi(\bar{p})$ can be presented as $\psi(\bar{p})=a_{1} \psi_{1}(\bar{p})+a_{2} \psi_{2}(\bar{p})$ for particles and $\psi^{\prime}(\bar{p})=a_{1}^{\prime} \psi_{1}^{\prime}(\bar{p})+a_{2}^{\prime} \psi_{2}^{\prime}(\bar{p})$ for antiparticles where $a_{1}, a_{2}, a_{1}^{\prime}, a_{2}^{\prime}$ are arbitrary complex parameters. Hence, the equation $Q \psi(\bar{p})=0$ in (4) generates eight homogeneous linear equations for $a_{1}, a_{2}, a_{1}^{\prime}, a_{2}^{\prime}$ and a nontrivial solution of these equations can exist only by special restriction on parameters $r_{k}$ of the matrix $Q$ and on the impulse of particles. We consider four equations for $a_{1}, a_{2}$ following from the equation $Q \psi(\bar{p})=a_{1} \psi_{1}(\bar{p})+a_{2} \psi_{2}(\bar{p})$ and obtain a nontrivial solution to them. The solution of four equations $\psi^{\prime}(\bar{p})=a_{1}^{\prime} \psi_{1}^{\prime}(\bar{p})+a_{2}^{\prime} \psi_{2}^{\prime}(\bar{p})$ can be obtained analogously. 


\section{Solution of equation $Q \psi(\bar{p})=0$}

Since $\tau_{0}^{( \pm)} Q=Q^{( \pm)}$and $\tau_{0}^{(+)}+\tau_{0}^{(-)}=1$, it follows from $Q \psi(\bar{p})=0$ that $Q^{( \pm)} \psi(\bar{p})=0$, and we obtain the following system of equations for $a_{1}, a_{2}$. The equation $Q^{(+)} \psi(\bar{p})=0$ is equivalent to

$$
\begin{gathered}
a_{1}\left(\left(m+p_{0}\right)\left(r_{18}^{+}-r_{45}^{-}\right)-p_{2}\left(i r_{27}^{+}+r_{36}^{-}\right)\right)-a_{2}\left(p_{1}-i p_{2}\right)\left(i r_{27}^{+}+r_{36}^{-}\right)=0, \\
a_{1}\left(\left(m+p_{0}\right)\left(i r_{27}^{+}-r_{36}^{-}\right)-p_{2}\left(r_{18}^{+}+r_{45}^{-}\right)\right)-a_{2}\left(p_{1}-i p_{2}\right)\left(r_{18}^{+}+r_{45}^{-}\right)=0
\end{gathered}
$$

and equation $Q^{(-)} \psi(\bar{p})=0$ reads

$$
\begin{array}{r}
a_{1}\left(-i p_{1}+p_{2}\right)\left(r_{27}^{-}+i r_{36}^{+}\right)+a_{2}\left(p_{2}\left(i r_{27}^{-}-r_{36}^{+}\right)+\left(m+p_{0}\right)\left(r_{18}^{-}+r_{45}^{+}\right)\right)=0, \\
a_{1}\left(p_{1}+i p_{2}\right)\left(r_{45}^{+}-r_{18}^{-}\right)+a_{2}\left(\left(m+p_{0}\right)\left(i r_{27}^{-}+r_{36}^{+}\right)+p_{2}\left(r_{18}^{-}-r_{45}^{+}\right)\right)=0 .
\end{array}
$$

Taking into account that $p_{0}>0$ and $p_{0}^{2}=p_{1}^{2}+p_{2}^{2}+p_{3}^{2}+m^{2}$, we obtain the following solvability conditions for three systems from two equations $(5-8)$.

For equations $(5,6)$ and $(7,8)$

$$
\begin{gathered}
\left(p_{1}-i p_{2}\right)\left(r_{18}^{+2}+r_{27}^{+2}+r_{36}^{-2}-r_{45}^{-2}\right)=0, \\
\left(p_{1}+i p_{2}\right)\left(r_{18}^{-2}+r_{27}^{-2}+r_{36}^{+2}-r_{45}^{+2}\right)=0,
\end{gathered}
$$

for equations $(5,7)$

$$
\begin{array}{r}
m\left(\left(i r_{27}^{+}+r_{36}^{-}\right)\left(i r_{27}^{-}-r_{36}^{+}\right)+\left(r_{18}^{+}-r_{45}^{-}\right)\left(r_{18}^{-}+r_{45}^{+}\right)\right)+ \\
+p_{3}\left(\left(i r_{27}^{-}-r_{36}^{+}\right)\left(r_{18}^{+}-r_{45}^{-}\right)-\left(i r_{27}^{+}+r_{36}^{-}\right)\left(r_{18}^{-}+r_{45}^{+}\right)\right)- \\
-p_{0}\left(\left(i r_{27}^{+}+r_{36}^{-}\right)\left(i r_{27}^{-}-r_{36}^{+}\right)-\left(r_{18}^{+}-r_{45}^{-}\right)\left(r_{18}^{-}+r_{45}^{+}\right)\right)=0 .
\end{array}
$$

Let us suppose that $p_{1}^{2}+p_{2}^{2}>0$. In this case, we obtain from (14), (15) the following conditions for parameters of the matrix $Q$ :

$$
r_{18}^{+2}+r_{27}^{+2}+r_{36}^{-2}-r_{45}^{-2}=0, r_{18}^{-2}+r_{27}^{-2}+r_{36}^{+2}-r_{45}^{+2}=0 \text {. }
$$

Since all of these parameters and $p_{0}, p_{3}, m$ are real numbers, the equation (11) is fulfilled if

$$
\begin{array}{r}
p_{0}=m \frac{A_{1}}{C_{1}}, p_{3}=m \frac{B_{1}}{C_{1}}, \\
A_{1}=\left(r_{27}^{+}\left(r_{18}^{+}-r_{45}^{-}\right)\left(r_{36}^{+2}+r_{27}^{-2}+\left(r_{18}^{-}+r_{45}^{+}\right)^{2}\right)-\right. \\
\left.-r_{27}^{-}\left(r_{18}^{-}+r_{45}^{+}\right)\left(\left(r_{18}^{+}-r_{45}^{-}\right)^{2}+r_{27}^{+2}+r_{36}^{-2}\right)\right), \\
B_{1}=2\left(r_{27}^{+} r_{36}^{+}-r_{27}^{-} r_{36}^{-}\right)\left(r_{18}^{+}-r_{45}^{-}\right)\left(r_{18}^{-}+r_{45}^{+}\right), \\
C_{1}=r_{27}^{+}\left(r_{18}^{+}-r_{45}^{-}\right)\left(r_{27}^{-2}+r_{36}^{+2}-\left(r_{18}^{-}+r_{45}^{+}\right)^{2}\right)- \\
-r_{27}^{-}\left(r_{18}^{-}+r_{45}^{+}\right)\left(r_{27}^{+2}+r_{36}^{-2}-\left(r_{18}^{+}-r_{45}^{-}\right)^{2}\right) .
\end{array}
$$

In virtue of (12), it follows from (13) that

$$
p_{0}=m \frac{r_{27}^{-} r_{45}^{-}+r_{27}^{+} r_{45}^{+}}{r_{18}^{+} r_{27}^{-}-r_{18}^{-} r_{27}^{+}}, p_{3}=m \frac{r_{27}^{+} r_{36}^{+}-r_{27}^{-} r_{36}^{-}}{r_{18}^{+} r_{27}^{-}-r_{18}^{-} r_{27}^{+}} .
$$

Taking into account that $p_{1}^{2}+p_{2}^{2}=p_{0}^{2}-p_{3}^{2}-m^{2}$ and using (14), we obtain

$$
p_{1}^{2}+p_{2}^{2}=\frac{2 m^{2} r_{27}^{-} r_{27}^{+}\left(r_{18}^{-} r_{18}^{+}+r_{27}^{-} r_{27}^{+}+r_{36}^{-} r_{36}^{+}+r_{45}^{-} r_{45}^{+}\right)}{\left(r_{18}^{+} r_{27}^{-}-r_{18}^{-} r_{27}^{+}\right)^{2}}
$$

with restriction for parameters of the matrix $Q$

$$
r_{27}^{-} r_{27}^{+}\left(r_{18}^{-} r_{18}^{+}+r_{27}^{-} r_{27}^{+}+r_{36}^{-} r_{36}^{+}+r_{45}^{-} r_{45}^{+}\right)>0 .
$$


Thus, if $\theta$ is the angle between the momentum vector $\vec{p}=\left(p_{1}, p_{2}, p_{3}\right)$ and the $x^{3}$-axis, then

$$
\tan ^{2}(\theta)=\frac{2 r_{27}^{-} r_{27}^{+}\left(r_{18}^{-} r_{18}^{+}+r_{27}^{-} r_{27}^{+}+r_{36}^{-} r_{36}^{+}+r_{45}^{-} r_{45}^{+}\right)}{\left(r_{27}^{+} r_{36}^{+}-r_{27}^{-} r_{36}^{-}\right)^{2}} .
$$

Now, we consider the equations (5-8) in the situation $p_{1}=p_{2}=0$. In this case they are written as

$$
\begin{array}{r}
a_{1}\left(p_{3}\left(i r_{27}^{+}+r_{36}^{-}\right)-\left(m+p_{0}\right)\left(r_{18}^{+}-r_{45}^{-}=0,\right.\right. \\
a_{1}\left(\left(m+p_{0}\right)\left(i r_{27}^{+}-r_{36}^{-}\right)-p_{3}\left(r_{18}^{+}+r_{45}^{-}\right)\right)=0, \\
a_{2}\left(p_{3}\left(i r_{27}^{-}-r_{36}^{+}\right)+\left(m+p_{0}\right)\left(r_{18}^{-}+r_{45}^{+}\right)\right)=0, \\
a_{2}\left(\left(m+p_{0}\right)\left(i r_{27}^{-}+r_{36}^{+}\right)+p_{3}\left(r_{18}^{-}-r_{45}^{+}\right)\right)=0 .
\end{array}
$$

Taking into account that $p_{0}^{2}=p_{3}^{2}+m^{2}$ by $p_{1}=p_{2}=0$, we obtain the following results. There is solution of the equations $(18-21)$ with arbitrary $a_{1} \neq 0$ and $a_{2}=0$, if

$$
\begin{array}{r}
\text { 1) } r_{27}^{+}=0, r_{18}^{+2}+r_{36}^{-2}=r_{45}^{-2}, r_{36}^{-} \neq 0, r_{18}^{+}+r_{45}^{-} \neq 0, r_{45}^{-} r_{18}^{+}>0, \\
p_{3}=\frac{\left(m+p_{0}\right)\left(r_{18}^{+}-r_{45}^{-}\right)}{r_{36}^{-}}=-\frac{\left(m+p_{0}\right) r_{36}^{-}}{r_{18}^{+}+r_{45}^{-}}=-\frac{m r_{36}^{-}}{r_{18}^{+}}, p_{0}=\frac{m r_{45}^{-}}{r_{18}^{+}} ; \\
\text {2) } r_{27}^{+}=0, r_{36}^{+}=0, r_{18}^{+}=r_{45}^{-}, p_{3}=0, p_{0}=m .
\end{array}
$$

There is solution of (18 - 21) with $a_{1}=0$ and arbitrary $a_{2} \neq 0$, if

$$
\begin{aligned}
& \text { 1) } r_{27}^{-}=0, r_{18}^{-2}+r_{36}^{+2}=r_{45}^{+2}, r_{36}^{-} \neq 0, r_{18}^{-}-r_{45}^{+} \neq 0, r_{45}^{+} r_{18}^{-}<0, \\
& p_{3}=\frac{\left(m+p_{0}\right)\left(r_{18}^{-}+r_{45}^{+}\right)}{r_{36}^{+}}=\frac{\left(m+p_{0}\right) r_{36}^{+}}{r_{45}^{+}-r_{18}^{-}}=-\frac{m r_{36}^{+}}{r_{18}^{-}}, p_{0}=-\frac{m r_{45}^{+}}{r_{18}^{-}} ; \\
& \text {2) } r_{27}^{-}=0, r_{36}^{-}=0, r_{18}^{-}+r_{45}^{+}=0, p_{3}=0, p_{0}=m .
\end{aligned}
$$

There is solution of (18 - 21) with arbitrary $a_{1} \neq 0$ and $a_{2} \neq 0$, if

$$
\begin{aligned}
& \text { 1) } r_{27}^{+}=r_{27}^{-}=0, r_{18}^{-2}+r_{36}^{+2}=r_{45}^{+2}, r_{18}^{-2}+r_{36}^{+2}=r_{45}^{+2}, r_{45}^{-} r_{18}^{+}>0, r_{45}^{+} r_{18}^{-}<0 \text {, } \\
& \frac{r_{45}^{-}}{r_{18}^{+}}=-\frac{r_{45}^{+}}{r_{18}^{-}}, \frac{r_{36}^{+}}{r_{18}^{-}}=\frac{r_{36}^{-}}{r_{18}^{+}}, r_{36}^{-} \neq 0, r_{36}^{+} \neq 0, r_{18}^{-}-r_{45}^{+} \neq 0, r_{18}^{+}+r_{45}^{-} \neq 0, \\
& p_{0}=\frac{m r_{45}^{-}}{r_{18}^{+}}=-\frac{m r_{45}^{+}}{r_{18}^{-}}, p_{3}=-\frac{m r_{36}^{+}}{r_{18}^{-}}=-\frac{m r_{36}^{-}}{r_{18}^{+}} ; \\
& \text {2) } r_{27}^{-}=r_{27}^{+}=r_{36}^{-}=r_{36}^{+}=0, r_{18}^{+}+r_{45}^{-}=0, r_{18}^{-}-r_{45}^{+}=0, p_{3}=0, p_{0}=m \text {. }
\end{aligned}
$$

\section{Simple example}

Let us chose the parameters of the matrix $Q$ as follows:

$$
r_{18}^{-}=1, r_{18}^{+}=2, r_{27}^{-}=2, r_{27}^{+}=1, r_{36}^{-}=2, r_{36}^{+}=2, r_{45}^{-}=3, r_{45}^{+}=3 \text {. }
$$

Then the equations (12) are satisfied, and (14), (15), (17) read

$$
p_{0}=3 m, p_{3}=-\frac{2 m}{3}, p_{1}^{2}+p_{2}^{2}=\frac{68 m^{2}}{9}, \tan ^{2}(\theta)=17 .
$$

The inequality (16) is obviously fulfilled. Substituting in (5-8) the parameters (22) and the values of $p_{0}, p_{3}$ from (23) we obtain the system of equation for $a_{1}, a_{2}$

$$
\begin{array}{r}
(2 i-8) a_{1} m-(3-6 i) a_{2}\left(i p_{1}+p_{2}\right)=0, \\
(12 i-14) a_{1} m-15 a_{2}\left(p_{1}-i p_{2}\right)=0, \\
(52-4 i) a_{2} m+(6+6 i) a_{1}\left(-i p_{1}+p_{2}\right)=0, \\
(28+24 i) a_{2} m+6 a_{1}\left(p_{1}+i p_{2}\right)=0 .
\end{array}
$$


The solution $a_{2}=(12 i-14) a_{1} /\left(15\left(p_{1}-I p_{2}\right)\right)$ of equations (24) fulfills also the equation (25) and $a_{2}=(1530+1785 i) a_{1}\left(i p_{1}-p_{2}\right) /(14450 m)$ is the solution of equations (26),(27). Since

$$
\begin{aligned}
& \frac{(12 i-14)}{\left(15\left(p_{1}-i p_{2}\right)\right)}-\frac{(1530+1785 i)\left(i p_{1}-p_{2}\right)}{14450 m}= \\
& =\frac{(595-510 i)\left(68 m^{2}-9\left(p_{1}^{2}+p_{2}^{2}\right)\right)\left(p_{1}+i p_{2}\right)}{43350 m\left(p_{1}^{2}+p_{2}^{2}\right)}
\end{aligned}
$$

and $68 m^{2}-9\left(p_{1}^{2}+p_{2}^{2}\right)=0$ in virtue of (23), the solutions of all the equations (24-27) are the same.

The spinor $\psi(\bar{p})$ for parameters $(26)$ is written in the form

$$
\psi(\bar{p})=\frac{4 a_{1} m}{15\left(p_{1}-i p_{2}\right)\left(m+p_{0}\right)}\left\{\begin{array}{c}
15\left(p_{1}-i p_{2}\right) \\
(12 i-14) m \\
(6-3 i)\left(p_{1}-i p_{2}\right) \\
(-26-2 i) m
\end{array}\right\}
$$

\section{Conclusions}

We have shown that in the model we used, the problem posed is exactly solvable. It turns out that there exist such sets of parameters of the matrix Q (characterizing the material properties of the plane interacting with the spinor field) for which a Dirac particle with the corresponding momentum and polarization freely flies through this plane. However, there are also such sets of parameters of the matrix Q for which this it is not possible with any momenta and polarizations of the particle. These features of the interaction of a particle with a plane are completely analogous to a locked door in a wall. If there is a key to the lock, then the door does not present an obstacle to pass through the wall, but without a suitable key passage is not possible. However, there are also walls without any doors, and no keys exist to facilitate passage through in this case. If 2D-materials with the properties for transparency to Dirac particles, as demonstrated by us in the framework of the mathematical model will be created, one can assume that they will find application both in scientific research and in technology.

\section{Acknowledgements}

The author is grateful to A.A.Andrianov for the fruitful discussion. This work was supported in part by Russian Foundation for Basic Research (Grant No 19-02-00983-a)

\section{References}

[1] K. Symanzik, Nucl.Phys. B 190, 1-44 (1981).

[2] N. N. Bogoliubov and D. V. Shirkov, Quantum Fields, Nauka, Moscow (1993);

C. Itzykson and J.-B. Zuber Quantum Fields Theory, McGraw-Hill, New York (1980).

[3] V. N. Markov and Yu. M. Pis'mak, J. Phys. A: Math. Gen. 39, 6525-6532 (2006); arXiv: hep-th/0505218v3 (2005).

[4] V. N. Marachevsky and Yu. M. Pis'mak, Phys. Rev. D 81, 065005 (2010); arXiv:0907.1985v2 [hep-th] (2009).

[5] D.Yu. Pis'mak and Yu. M. Pis'mak, Theor. Math. Phys. 175,816-826 (2013); Phys. Part. Nuclei 44, 450-461 (2013); Theor. Math. Phys. 166, 1423-1431 (2011).

[6] D.Yu. Pis'mak, Yu. M. Pis'mak and F. J. Wegner, Phys. Rev. E 92, 013204 (2015); arXiv:1406.1598v1 [hep-th] (2014). 
[7] D. Yu. Pismak and Yu. M. Pismak, Theor. Math. Phys. 184, 3, 1329-1341 (2015).

[8] D. Yu. Pismak and D. Yu. Shukhobodskaia, 126 EPJ Web of Conferences 05012 (2016) ICNFP 2015, 125 EPJ Web of Conferences 05022 (2016) QUARKS-2016

[9] Yu. M. Pismak, Phys. Part. Nucl. Lett, 15, 380-383 (2018)

[10] Yu. M. Pismak and O. Yu. Shakhova, Phys. Part. Nucl. Lett. 16, 441-444 (2019).

[11] F. J. Wegner and Yu. M. Pismak, Theor. and Math. Phys. 200, 1401-1412 (2019). 